ELECTRONIC RESEARCH ANNOUNCEMENTS OF THE AMERICAN MATHEMATICAL SOCIETY

Volume 3, Pages 121-125 (November 4, 1997)

S $1079-6762(97) 00036-\mathrm{X}$

\title{
WAVE PROPAGATION IN A LATTICE KPP EQUATION IN RANDOM MEDIA
}

\author{
TZONG-YOW LEE AND FRED TORCASO \\ (Communicated by Mark Freidlin)
}

\begin{abstract}
We extend a result of Freidlin and Gartner (1979) for KPP (Kolmogorov-Petrovskii-Piskunov) wave fronts to the case $d \geq 2$ for i.i.d. (independent and identically distributed) random media. We show a wave front propagation speed is attained for the discrete-space (lattice) KPP using a large deviation approach.
\end{abstract}

\section{INTRODUCTION}

The problem of wave front propagation in 1-dimensional space homogeneous random media was considered in Gartner and Freidlin (1979) [3], where they extend the results from the periodic case. See Freidlin (1985) [2] for an exposition. While the continuous space $R$ was used, their method and result extend to the lattice space $Z$ easily. The extension to higher dimensions $d>1$ presents some difficulty. We thank Mark Freidlin for suggesting this interesting problem. We will consider the lattice space $Z^{d}$ and assume that the random media at the lattice points are independent of one another. For an excellent account of analysis and modelling of front propagation in parabolic PDEs and their utility in applications see Xin (1997) [7].

In this paper we consider discrete-space KPP equations of the following form:

$$
\begin{gathered}
\frac{\partial u}{\partial t}=\tilde{\triangle} u+\xi(x) u(1-u), \quad t>0, \quad x \in Z^{d} \\
u(0, x)=1_{0}(x),
\end{gathered}
$$

where $\tilde{\triangle}$ is the discrete Laplacian: $\tilde{\triangle} f(x)=\frac{1}{2 d} \sum_{\mathbf{e}:|\mathbf{e}|}=1[f(x+\mathbf{e})-f(x)]$, and $1_{0}(x)=1$ if $x=0$ and equals 0 otherwise. The random field $\xi \equiv\left\{\xi(x): x \in Z^{d}\right\}$ is supported on the probability space $(\hat{\Omega}, \hat{\mathcal{F}}, \hat{P})$. It is not difficult to treat more general form of KPP nonlinearity. The above form is chosen for the simplicity of presentation. We further assume that the $\xi(x)$ are independent and identically distributed, bounded and nonnegative random variables, and we set

$$
A=\operatorname{ess} \sup \xi(0)
$$

Received by the editors June 20, 1997.

1991 Mathematics Subject Classification. Primary 60J60; Secondary 35K55.

Key words and phrases. KPP equation, random media, large deviations.

This work was supported under NSF Grant DMS-95-04177 while the second author was research assistant at the University of Maryland.

(C) 1997 American Mathematical Society 
where the ess sup is with respect to $\hat{P}$. The case where $\operatorname{ess} \sup \xi(0)=\infty$ is easy and is discussed in the concluding remark (iv). The solution $u(t, x)=u(t, x ; \hat{\omega})$ to (1) is a function of $t \geq 0, x \in Z^{d}$ and $\hat{\omega} \in \hat{\Omega}$.

In order to state the main result of this paper, we define some notation. We set for $z<-A$

$$
\mu(z ; \mathbf{e})=\lim _{t \rightarrow \infty} \frac{1}{t} \log E_{t \mathbf{e}}\left[e^{\int_{0}^{\tau_{0}}\left(\xi\left(\eta_{s}\right)+z\right) d s}\right] .
$$

Here $\eta$. is the strong Markov process on $Z^{d}$ that corresponds to the generator $\tilde{\triangle}$, a continuous time symmetric simple random walk, and $\tau_{a}$ is the first time $\eta_{t}$ hits the site $a \in Z^{d}$. We can prove that, if $z>-A$, then (2) is infinite under an assumption weaker than the i.i.d. assumption. It is an easy consequence of the subadditive ergodic theorem, see e.g. Liggett (1985) [4], that this limit exists $\hat{P}$-a.s., and since our underlying random media are ergodic, the limit $\mu(z ; \mathbf{e})$ is nonrandom. The limit function $\mu(z ; \mathbf{e})$ is also relevant in the study of Brownian motions in random potential and percolation processes. See Sznitman (1994) [5] for an excellent acccount of some relations and further references.

Let $I(y ; \mathbf{e})$ denote the Legendre transform of the function $\mu(z ; \mathbf{e})$ :

$$
I(y ; \mathbf{e})=\sup _{z<-A}[y z-\mu(z ; \mathbf{e})] .
$$

Probabilities and expectations with respect to the process $\eta$. will be denoted by $P_{x}$ and $E_{x}$, respectively. The subscript $x$ refers to the fact that the process $\eta$ starts at $x: \eta_{0}=x$. Since our functions evolve in $Z^{d}$, it is important that $x$ be in $Z^{d}$, so by $P_{x}$ and $E_{x}$ we will mean to use $x=[x]$, the nearest lattice site to $x$, where there is some deterministic rule to break ties. Similarly, $u(t, x)=u(t,[x])$.

The main result of this paper is

Theorem. Make assumptions as above and let $\mathbf{e} \in R^{d} \backslash\{0\}$. Then for any $v>0$, $\hat{P}$-a.s.,

$$
\lim _{t \rightarrow \infty} \frac{1}{t} \log u(t, t v \mathbf{e})=-\left[v I\left(\frac{1}{v} ; \mathbf{e}\right) \vee 0\right]
$$

where $a \vee b=\max (a, b)$. Moreover, $I\left(\frac{1}{v} ; \mathbf{e}\right)=0$ has a unique solution $v=v_{\mathbf{e}}$ which can be characterized as

$$
v_{\mathbf{e}}=\inf _{z<-A} \frac{z}{\mu(z ; \mathbf{e})} .
$$

Remark 1. The i.i.d. assumption on $\xi$ is used only in one place in the proof. Elsewhere it is replaced by the weaker assumption of ergodic stationary media of "purely" random type, by which we mean that $\hat{P}$ satisfies

$$
\text { For any } \begin{aligned}
L> & 0 \text { and } \varepsilon>0, \\
& \hat{P}\left[\xi(x)>\operatorname{ess} \sup \xi(0)-\varepsilon, \forall x \in \Lambda_{L}\right]>0,
\end{aligned}
$$

where $\Lambda_{L}$ is the box centered at the origin of side width $2 L+1$.

Note that a periodic medium is not purely random. Corresponding wave propagation results for periodic media can be found in Freidlin (1985) [2]. See his Section 7.3 for the case of arbitrary dimensions. For the one-dimensional case he showed that the periodic case is a special case subject to his result for random media (see Sections 7.4-7). We believe that the above theorem holds for all purely random media, but do not have a proof. It would be interesting to see a unified approach worked out for both periodic and purely random cases in high dimensions $(d \geq 2)$. 
Remark 2. In view of this theorem we define $v_{\mathbf{e}}$ as the wave speed in the logarithmic sense. The variational formula of $v_{\mathbf{e}}$ is easily derived from the definition of $I$. The speed $v_{\mathbf{e}}$ is nonrandom as $\mu(z ; \mathbf{e})$ is. We believe that the solution $u$ goes to $1\left(\hat{P}_{-}\right.$ a.s.) for $v<v_{\mathbf{e}}$ and do not work on its proof. Such a result should follow from analysis similar to that in Freidlin (1985, p. 521) [2].

Let us summarize some basic properties of the functions $\mu$ and $I$ in the following

Lemma. Let $\mathbf{e} \in R^{d} \backslash\{0\}$.

(a) The function $\mu(z ; \mathbf{e})=\lim _{t \rightarrow \infty} \frac{1}{t} \log E_{t \mathbf{e}}\left[e^{\int_{0}^{\tau_{0}} \xi\left(\eta_{s}\right)+z d s}\right]>-\infty$ exists $\hat{P}$ a.s., is a convex function in $z$, and $a \mu(z ; \mathbf{e})=\mu(z ; a \mathbf{e})$.

(b) $\mu(z ; \mathbf{e})$ is a concave function of $\mathbf{e}$.

(c) $\mu(z ; \mathbf{e})$ tends to $-\infty$ as $z$ tends to $-\infty$.

(d) The Legendre transform $I(y ; \mathbf{e})($ see $(3))$ of $\mu(z ; \mathbf{e})$ defined above is a convex function of both its arguments, is a strictly decreasing function of $y>0$, and $I(y ; \mathbf{e})$ tends to $\infty$ as $y \downarrow 0$.

(e) The exponent $v I\left(\frac{1}{v} ; \mathbf{e}\right)$ in the main theorem equals $I(1 ; v \mathbf{e})$ and is convex in $\mathbf{f}=v \mathbf{e}$, hence in $v$ for fixed $\mathbf{e}$.

(f) The wave speed $v_{\mathbf{e}}$ of this paper is unique.

We should say a few words about the proof of the main result. That $u \leq 1$ is readily seen from a comparison if we substitute the initial function in (1) by the function identically equal to 1 . This explains why the logarithmic limit is nonpositive in the Theorem. As can be guessed, the upper bound utilizes (2), the limit of a certain cumulant generating function, and a Chebyshev type of inequality. The details follow the steps of Gartner and Freidlin (1979) [3] closely.

For the lower bound, a difficulty arises that does not appear in the one-dimensional case studied in Gartner and Freidlin (1979) [3]. In that 1-d case, $\mu(z)$ is in fact a smooth function of $z$ (this follows from the ergodic theorem), and we can easily apply the Gartner-Ellis theorem (e.g. Dembo and Zeitouni (1993) [1]). However, in the case of $d \geq 2$ we only know the function $\mu(z)$ is convex, so there needs to be more work to derive a useful large deviation lower bound. We are able to obtain the good lower bound, using essentially the idea of Lemma 20 in Zerner (1997) [8]. In Zerner's proof he uses first passage percolation times to help prove a lower bound large deviation result. We basically illustrate the parts of his proof that we need without the use of first passage percolation times.

\section{REMARKS}

We conclude this article with some remarks.

(i) Let $v_{\mathbf{e}}$ be the wave speed in the direction $\mathbf{e}$ and let $\delta>0$ be arbitrary. Let $C_{\mathbf{e}, \delta}$ be the cone region

$$
C_{\mathbf{e}, \delta}=\{a \mathbf{f}: a>0,|\mathbf{f}-\mathbf{e}|<\delta\} .
$$

Then the wave speed remains unchanged if we decrease $\xi(x)$ for $x \notin C_{\mathbf{e}, \delta}$, as both the upper and lower bounds remain effective. Clearly, the upper bound holds. The lower bound holds, due to the fact that the trajectories "wandering out of the cone $C_{\mathbf{e}, \delta}$ " did not contribute at all.

(ii) What happens to the wave speed $v_{\mathbf{e}}$ if we increase $\xi(x)$ for $x \notin C_{\mathbf{e}, \delta}$ ? The speed $v_{\mathbf{e}}$ can get a real boost. For example, for $x \notin C_{\mathbf{e}, \delta}$ let a large $B>1$ be multiplied by the original $\xi(x)$ : we have $B \xi(x)$ for $x \notin C_{\mathbf{e}, \delta}$. In any direction $\mathbf{b}$ 
outside of $\overline{C_{\mathbf{e}, \delta}}$, the closure of the cone, (i) then implies that $v_{\mathbf{b}}$ is as in the case of $B \xi(x)$ for $x \in Z^{d}$ random media, which is as large as one desires (by choosing large $B)$. It is now easy to see that the most favorable trajectory, in the case of large $B$, would be wandering out of the cone $C_{\mathbf{e}, \delta}$ first and then take advantage of the enhanced (by large $B$ ) wave speed $v_{\mathbf{b}}$.

(iii) It is interesting to know if $\mu(z ; \mathbf{e})$ is in fact a smooth function of $z$. As mentioned in the Introduction this is known to be true for 1-dimensional random media. For the case of higher-dimensional random media this is still unknown. If $\mu(z ; \mathbf{e})$ is a smooth function then the proof of the lower bound estimate is greatly simplified. There can be many approaches to showing such a result, but we would like to mention one. For instance, one can show the smoothness of $\mu(z)$ by showing the following variance bound:

$$
V^{Q_{t \mathbf{e}, z}}\left[\tau_{0}\right]=O(t)
$$

The smoothness of $\mu$ would then follow from the Arzéla-Ascoli theorem. Sznitman (1995) showed that $0<\lim \sup _{t \rightarrow \infty} \frac{1}{t} E^{Q_{t \mathbf{e}, z}}\left[\tau_{0}\right]<\infty$. It would be interesting to see a central limit theorem proved or disproved $\hat{P}$-a.s. for the (standardized) $\tau_{0}$ with respect to the tilted measure $Q_{t \mathbf{e}, z}, z<-A$.

(iv) As a last remark we would like to say something in the case where $\xi(0)$ is an unbounded nonnegative random variable. It turns out that the speed $v=v_{\mathbf{e}}$ will be infinite for all $\mathbf{e} \in R^{d} \backslash\{0\}$. To see this let $W_{(k)}$ be the truncation of $\xi(0)$ at $k, k \geq 0: W_{(k)}=\xi(0)$ if $\xi(0) \leq k$, and $W_{(k)}=k$ if $\xi(0)>k$. Let $v_{(k)}$ and $\mu_{(k)}(z)$ be the wave front speed and $\mu$-function, respectively, associated with i.i.d. $W_{(k)}$-distributed random media. Here, since we fix an $\mathbf{e} \in R^{d} \backslash\{0\}$, we omit writing the $\mathbf{e}$ in our notation. Clearly $v \geq v_{(k)}$ and both $v_{(k)}$ and $\mu_{(k)}$ are nondecreasing in $k$. The main theorem gives

$$
v \geq v_{(k)}=\inf _{z<-k} \frac{z}{\mu_{(k)}(z)} \geq \inf _{z<-k} \frac{z}{\mu_{(0)}(z)} .
$$

Now $\mu_{(0)}$ can be estimated in all dimensions. We will demonstrate $v=\infty$ only in the 1-dimensional case. In this case, we have an explicit formula for $\mu_{(0)}(z)$ which can be easily shown to be

$$
\mu_{(0)}(z)=-2 \sinh ^{-1} \sqrt{-\frac{z}{2}}
$$

The right-hand side of (5) is then readily seen to tend to infinity as $k$ tends to infinity.

\section{ACKNOWLEDGEMENTS}

We would like to thank David Edwards for many useful conversations and AlainSol Sznitman for giving us his papers. We would like to thank Martin Zerner for his valuable comments during his visit with us, and highlighting the important concepts in his work. We would also like to thank Mark Freidlin for his support and interest in this problem.

\section{REFERENCES}

[1] Dembo, A. and Zeitouni, O. (1993), Large deviation techniques and applications, Jones and Bartlett Publishers, Boston, MA. MR 95a:60034

[2] Freidlin, M. I. (1985), Functional integration and partial differential equations, Annals of Mathematical Studies, vol. 109, Princeton University Press. MR 87g:60066 
[3] Gartner, J. and Freidlin, M. I. (1979), On the propagation of concentration waves in periodic and random media, Dokl. Akad. Nauk SSSR 249, 521-525. (Russian) MR 81d:80005

[4] Liggett, Thomas (1985), Interacting particle systems, Springer-Verlag. MR 86e:60089

[5] Sznitman, A. S. (1994), Shape theorem, Lyapounov exponents, and large deviations for Brownian motion in a Poissonian potential, Comm. Pure Appl. Math. 47, 1655-1688. MR 96b:60217

[6] Sznitman, A. S. (1995), Crossing velocities and random lattice animals, Ann. Probab. 23, 1006-1023. MR 96j:60066

[7] Xin, Jack X. (1997), Analysis and modeling of front propagation in heterogeneous media, To appear in Surveys in Applied Math.

[8] Zerner, Martin (1997), Directional decay of the Green's function for a random nonnegative potential on $Z^{d}$, To appear in Annals of Applied Probability.

Department of Mathematics, University of Maryland, College Park, MD 20742

E-mail address: tyl@math.umd.edu

Department of Mathematics, University of Maryland, College Park, MD 20742

E-mail address: torcaso@math.umd.edu 